\title{
Research Article \\ Schauder Basis, Separability, and Approximation Property in Intuitionistic Fuzzy Normed Space
}

\author{
M. Mursaleen, ${ }^{1}$ V. Karakaya, ${ }^{2}$ and S. A. Mohiuddine ${ }^{3}$ \\ ${ }^{1}$ Department of Mathematics, Aligarh Muslim University, Aligarh 202002, India \\ ${ }^{2}$ Department of Mathematical Engineering, Yildiz Technical University, Davutpasa Campus, \\ Esenler, Istanbul 34220, Turkey \\ ${ }^{3}$ Department of Mathematics, Faculty of Science, Jazan University, Jazan 45142, Saudi Arabia \\ Correspondence should be addressed to M. Mursaleen, mursaleenm@gmail.com
}

Received 23 August 2010; Accepted 30 November 2010

Academic Editor: Norimichi Hirano

Copyright (C 2010 M. Mursaleen et al. This is an open access article distributed under the Creative Commons Attribution License, which permits unrestricted use, distribution, and reproduction in any medium, provided the original work is properly cited.

We define and study the concepts of Schauder basis, separability, and approximation property in intuitionistic fuzzy normed spaces and establish some results related to these concepts. We also display here some interesting examples by using classical sequence spaces $\ell_{p}(1 \leq p \leq \infty)$.

\section{Introduction and Background}

We will write $w$ for the set of all complex sequences $x=\left(x_{k}\right)_{k=0}^{\infty}$. Let $\ell_{\infty}, c$, and $c_{0}$ denote the sets of all bounded, convergent, and null sequences, respectively. We write $\ell_{p}:=\{x \in w$ : $\left.\sum_{k=0}^{\infty}\left|x_{k}\right|^{p}<\infty\right\}$ for $1 \leq p<\infty$. By $e$ and $e^{(n)}(n \in \mathbb{N})$, we denote the sequences such that $e_{k}=1$ for $k=0,1, \ldots, e_{n}^{(n)}=1$, and $e_{k}^{(n)}=0(k \neq n)$. Note that $c_{0}, c$, and $\ell_{\infty}$ are Banach spaces with the sup-norm $\|x\|_{\infty}=\sup _{k}\left|x_{k}\right|$, and $\ell_{p}(1 \leq p<\infty)$ are Banach spaces with the norm $\|x\|_{p}=\left(\sum\left|x_{k}\right|^{p}\right)^{1 / p}$.

A sequence $\left(b^{(n)}\right)_{n=0}^{\infty}$ in a normed linear space $X$ is called a Schauder basis [1] if for every $x \in X$, there is a unique sequence $\left(\beta_{n}\right)_{n=0}^{\infty}$ of scalars such that $x=\sum_{n=0}^{\infty} \beta_{n} b^{(n)}$, that is, $\lim _{m \rightarrow \infty}\left\|x-\sum_{n=0}^{m} \beta_{n} b^{(n)}\right\|_{X}=0$.

Recently, the concept of intuitionistic fuzzy normed space has been introduced and studied by Saadati and Park [2] and further studied by Mursaleen and Mohiuddine [3-5]. The concept of Schauder basis and its applications have recently been studied by Palomares et al. [6] and by Yilmaz [7]. In this paper, we define and study the concept of Schauder basis, separability, and approximation property in intuitionistic fuzzy normed spaces and establish some results related to these concepts analogous to those of Yilmaz [7]. We also display here some interesting examples by using classical sequence spaces $\ell_{p}(1 \leq p \leq \infty)$. 
In this section, we recall some notations and basic definitions used in this paper.

Definition 1.1. A binary operation $*:[0,1] \times[0,1] \rightarrow[0,1]$ is said to be a continuous t-norm if it satisfies the following conditions:

(a) $*$ is associative and commutative,

(b) $*$ is continuous,

(c) $a * 1=a$ for all $a \in[0,1]$,

(d) $a * b \leq c * d$ whenever $a \leq c$ and $b \leq d$ for each $a, b, c, d \in[0,1]$.

Definition 1.2. A binary operation $\diamond:[0,1] \times[0,1] \rightarrow[0,1]$ is said to be a continuous $t$-conorm if it satisfies the following conditions:

$\left(a^{\prime}\right) \diamond$ is associative and commutative,

$\left(b^{\prime}\right) \diamond$ is continuous,

$\left(c^{\prime}\right) a \diamond 0=a$ for all $a \in[0,1]$,

$\left(d^{\prime}\right) a \diamond b \leq c \diamond d$ whenever $a \leq c$ and $b \leq d$ for each $a, b, c, d \in[0,1]$.

Using the notions of continuous t-norm and $t$-conorm, Saadati and Park [2] have recently introduced the concept of intuitionistic fuzzy normed space as follows.

Definition 1.3. The five-tuple $(X, \mu, v, *, \diamond)$ is said to be an intuitionistic fuzzy normed spaces (for short, IFNS) if $X$ is a vector space, $*$ is a continuous $t$-norm, $\diamond$ is a continuous $t$-conorm, and $\mu, v$ are fuzzy sets on $X \times(0, \infty)$ satisfying the following conditions. For every $x, y \in X$ and $s, t>0$,

(i) $\mu(x, t)+v(x, t) \leq 1$,

(ii) $\mu(x, t)>0$,

(iii) $\mu(x, t)=1$ if and only if $x=0$,

(iv) $\mu(\alpha x, t)=\mu(x, t /|\alpha|)$ for each $\alpha \neq 0$,

(v) $\mu(x, t) * \mu(y, s) \leq \mu(x+y, t+s)$,

(vi) $\mu(x, \cdot):(0, \infty) \rightarrow[0,1]$ is continuous,

(vii) $\lim _{t \rightarrow \infty} \mu(x, t)=1$ and $\lim _{t \rightarrow 0} \mu(x, t)=0$,

(viii) $v(x, t)<1$,

(ix) $v(x, t)=0$ if and only if $x=0$,

(x) $v(\alpha x, t)=v(x, t /|\alpha|)$ for each $\alpha \neq 0$,

(xi) $v(x, t) \diamond v(y, s) \geq v(x+y, t+s)$,

(xii) $v(x, \cdot):(0, \infty) \rightarrow[0,1]$ is continuous, and

(xiii) $\lim _{t \rightarrow \infty} \mathcal{v}(x, t)=0$ and $\lim _{t \rightarrow 0} v(x, t)=1$.

In this case $(\mu, v)$ is called an intuitionistic fuzzy norm. 
Example 1.4. Let $(X,\|\cdot\|)$ be a normed space, $a * b=a b$, and $a \diamond b=\min \{a+b, 1\}$ for all $a, b \in[0,1]$. For all $x \in X$ and every $t>0$, consider

$$
\mu(x, t)=\left\{\begin{array}{ll}
\frac{t}{t+\|x\|} & \text { if } t>0, \\
0 & \text { if } t \leq 0 ;
\end{array} \quad v(x, t)= \begin{cases}\frac{\|x\|}{t+|x|} & \text { if } t>0 \\
1 & \text { if } t \leq 0 .\end{cases}\right.
$$

Then $(X, \mu, \nu, *, \diamond)$ is an IFNS.

Remark 1.5 (see [3]). Let $(X, \mu, v, *, \diamond)$ be an IFNS with the condition

$$
\mu(x, t)>0, \quad v(x, t)<1 \quad \text { implies } x=0 \forall t \in \mathbb{R} .
$$

Let $\|x\|_{\alpha}=\inf \{t>0: \mu(x, t) \geq \alpha$ and $v(x, t) \leq 1-\alpha\}$, for all $\alpha \in(0,1)$. Then $\left\{\|\cdot\|_{\alpha}: \alpha \in(0,1)\right\}$ is an ascending family of norms on $X$. These norms are called $\alpha$-norms on $X$ corresponding to intuitionistic fuzzy norm $(\mu, v)$.

\section{Some Topological Concepts in IFNS}

Recently, the strong and weak intuitionistic fuzzy convergence as well as strong and weak intuitionistic fuzzy limit were discussed by Mursaleen and Mohiuddine [3].

Definition 2.1. Let $(X, \mu, v, *, \diamond)$ be an IFNS. Then, a sequence $\left(x_{k}\right)$ is said to be

(i) weakly intuitionistic fuzzy convergent to $x \in X$ if and only if, for every $\epsilon>0$ and $\alpha \in(0,1)$, there exists some $k_{0}=k_{0}(\alpha, \epsilon)$ such that $\mu\left(x_{k}-x, \epsilon\right) \geq 1-\alpha$ and $v\left(x_{k}-\right.$ $x, \epsilon) \leq \alpha$ for all $k \geq k_{0}$. In this case we write $x_{k} \stackrel{\text { wif }}{\longrightarrow} x$,

(ii) strongly intuitionistic fuzzy convergent to $x \in X$ if and only if, for every $\alpha \in(0,1)$, there exists some $k_{0}=k_{0}(\alpha)$ such that $\mu\left(x_{k}-x, t\right) \geq 1-\alpha$ and $v\left(x_{k}-x, t\right) \leq \alpha$ for all $t>0$. In this case we write $x_{k} \stackrel{\text { sif }}{\longrightarrow} x$.

The following result characterizes the (wif)- and (sif)-limit through $\alpha$-norms.

Proposition 2.2. Let $\left(X, \mu_{1}, \nu_{1}, *, \diamond\right)$ and $\left(Y, \mu_{2}, \nu_{2}, *, \diamond\right)$ be two IFNS satisfying (1.2) and $f: X \rightarrow$ $Y$ be a mapping. Then

(i) (wif)- $\lim _{\mathrm{x} \rightarrow \mathrm{x}_{0}} \mathrm{f}(\mathrm{x})=\mathrm{L}$ if and only if, for each $\alpha \in(0,1)$

$$
\lim _{\left\|x-x_{0}\right\|_{\alpha}^{(1)} \rightarrow 0}\|f(x)-L\|_{\alpha}^{(2)}=0
$$

(ii) (sif) $\lim _{\mathrm{x} \rightarrow \mathrm{x}_{0}} \mathrm{f}(\mathrm{x})=\mathrm{L}$ if and only if

$$
\lim _{\left\|x-x_{0}\right\|_{\alpha}^{(1)} \rightarrow 0}\|f(x)-L\|_{\alpha}^{(2)}=0 \quad \text { uniformly in } \alpha,
$$

where $\|\cdot\|_{\alpha}^{(1)}$ and $\|\cdot\|_{\alpha}^{(2)}$ are $\alpha$-norms of the intuitionistic fuzzy norms $\left(\mu_{1}, v_{1}\right)$ and $\left(\mu_{2}, v_{2}\right)$, respectively. 
Proof. Here we prove the case (ii). Suppose that (sif)- $\lim _{x \rightarrow x_{0}} f(x)=L$. For a given $\epsilon>0$, there exists some $\delta=\delta(\epsilon)>0$ such that

$$
\mu_{2}(f(x)-L, \epsilon) \geq \mu_{1}\left(x-x_{0}, \delta\right), \quad v_{2}(f(x)-L, \epsilon) \geq v_{1}\left(x-x_{0}, \delta\right),
$$

for all $x \in X$. For each $\alpha \in(0,1)$, if

$$
\left\|x-x_{0}\right\|_{\alpha}^{(1)}=\inf \left\{t>0: \mu_{1}\left(x-x_{0}, t\right) \geq \alpha, v_{1}\left(x-x_{0}, t\right) \leq 1-\alpha\right\}<\delta,
$$

then $\mu_{1}\left(x-x_{0}, \delta\right) \geq \alpha$ and $v_{1}\left(x-x_{0}, \delta\right) \leq 1-\alpha$. Hence $\mu_{2}(f(x)-L, \epsilon) \geq \alpha$ and $v_{2}(f(x)-L, \epsilon) \leq 1-\alpha$ and so that $\|f(x)-L\|_{\alpha}^{(2)}<\epsilon$. Since $\delta$ does not depend on $\alpha$, we get

$$
\lim _{\left\|x-x_{0}\right\|_{\alpha}^{(1)} \rightarrow 0}\|f(x)-L\|_{\alpha}^{(2)}=0 \quad \text { uniformly in } \alpha .
$$

Conversely, let $\lim _{\left\|x-x_{0}\right\|_{\alpha}^{(1)} \rightarrow 0}\|f(x)-L\|_{\alpha}^{(2)}=0$ uniformly in $\alpha$. Given $\epsilon>0$, there exists some $\delta=\delta(\epsilon)>0$ such that

$$
\left\|x-x_{0}\right\|_{\alpha}^{(1)}<\delta \quad \text { implies }\|f(x)-L\|_{\alpha}^{(2)}<\epsilon
$$

for all $x \in X$ and $\alpha \in(0,1)$. Choose some $\lambda<\mu_{1}\left(x-x_{0}, \delta\right)$ and $\lambda>v_{1}\left(x-x_{0}, \delta\right)$ or $v_{1}\left(x-x_{0}, \delta\right)<$ $\lambda<\mu_{1}\left(x-x_{0}, \delta\right)$. Since

$$
\mu_{1}\left(x-x_{0}, \delta\right)=\sup \left\{\alpha \in(0,1):\left\|x-x_{0}\right\|_{\alpha}^{(1)}<\delta\right\}
$$

there exists some $\alpha_{0} \in(0,1)$ such that $\lambda<\alpha_{0}$ and $\left\|x-x_{0}\right\|_{\alpha_{0}}^{(1)}<\delta$. Hence $\|f(x)-L\|_{\alpha_{0}}^{(2)}<\epsilon$ by the hypothesis, that is,

$$
\mu_{2}(f(x)-L, \epsilon) \geq \alpha_{0}>\lambda, \quad v_{2}(f(x)-L, \epsilon) \leq 1-\alpha_{0}<1-\lambda .
$$

So, we get $\mu_{2}(f(x)-L, \epsilon) \geq \mu_{1}\left(x-x_{0}, \delta\right)$ and $v_{2}(f(x)-L, \epsilon) \leq \mu_{1}\left(x-x_{0}, \delta\right)$.

Proposition 2.3. Let $\left(x_{k}\right)$ be a sequence in the IFNS $\left(X, \mu_{1}, v_{1}, *, \diamond\right)$ satisfying (1.2). Then

(i) $x_{k} \stackrel{\text { wif }}{\longrightarrow} x$ if and only if, for each $\alpha \in(0,1)$

$$
\lim _{k \rightarrow \infty}\left\|x_{k}-x\right\|_{\alpha}=0
$$

(ii) $x_{k} \stackrel{\text { sif }}{\longrightarrow} x$ if and only if

$$
\lim _{k \rightarrow \infty}\left\|x_{k}-x\right\|_{\alpha}=0 \quad \text { uniformly in } \alpha,
$$

where $\|\cdot\|_{\alpha}$ are $\alpha$-norms of the intuitionistic fuzzy norms $(\mu, v)$. 
The proof of the above theorem directly follows from Propositions 2.2.

We define the following concepts analogous to that of Yilmaz [7].

Definition 2.4. The sif(wif)-closure of a subset $B$ in IFNS $(X, \mu, v, *, \diamond)$ is the set of all $x \in X$ such that there exists a sequence $\left(x_{n}\right) \in B$ such that $x_{n} \stackrel{\text { sif(wif) }}{\longrightarrow} x$. In this case, we write $\bar{B}^{\text {sif }}\left(\bar{B}^{\text {wif }}\right)$. $B$ is said to be sif(wif)-closed whenever $\bar{B}^{\text {sif }}\left(\bar{B}^{\text {wif }}\right)=B$.

It is easy to see that $\bar{B}^{\text {sif }} \subseteq\left(\bar{B}^{\text {wif }}\right)$. The following example shows that inclusion is strict.

Example 2.5. Let $X=\mathbb{R}$ and

$$
\mu(x, t)=\left\{\begin{array}{ll}
\frac{t-|x|}{t+|x|} & \text { if } t>|x|, \\
0 & \text { if } t \leq|x| ;
\end{array} \quad v(x, t)= \begin{cases}\frac{2|x|}{t+|x|} & \text { if } t>|x|, \\
1 & \text { if } t \leq|x|\end{cases}\right.
$$

on $X$. Let $U_{X}=\{x \in X:|x|<1\}$ and we show that $\bar{U}_{X}^{\text {wif }}=B_{X}=\{x \in X:|x| \leq 1\}$. For every $x \in B_{X}$, there exists a sequence $\left(x_{n}\right) \subset U_{X}$ such that $\left\|x_{n}-x\right\|_{\alpha} \rightarrow 0$ as $n \rightarrow \infty$, for each $\alpha \in(0,1)$. This is accomplished by taking $x_{n}=(1-1 /(n+1)) x$ since each $x_{n} \in U_{X}$ and

$$
\left\|x_{n}-x\right\|_{\alpha}=\frac{1+\alpha}{1-\alpha}\left|x_{n}-x\right|<\left(\frac{1+\alpha}{1-\alpha}\right) \frac{1}{n+1} \longrightarrow 0 \text { as } n \longrightarrow \infty,
$$

for each $\alpha \in(0,1)$. However, $\bar{U}_{X}^{\text {sif }}=U_{X}$. Indeed for $x \in \bar{U}_{X}^{\text {sif }}$, there exists $\left(x_{n}\right) \subset U_{X}$ such that $\left\|x_{n}-x\right\|_{\alpha} \rightarrow 0$ as $n \rightarrow \infty$, uniformly in $\alpha$. This means that, given $\epsilon>0$, there exists an integer $n_{\circ}(\epsilon)>0$ such that for every $\alpha \in(0,1)$ and $n \geq n_{\circ}$,

$$
\left\|x_{n}-x\right\|_{\alpha}<\epsilon
$$

On the other hand,

$$
|x| \leq\left|x_{n}-x\right|+\left|x_{n}\right|<\left|x_{n}-x\right|+1=\left(\frac{1-\alpha}{1+\alpha}\right)\left\|x_{n}-x\right\|_{\alpha}+1<\left(\frac{1-\alpha}{1+\alpha}\right) \epsilon+1,
$$

for all $\alpha \in(0,1)$ and $n \geq n_{\circ}$. Letting $\epsilon \rightarrow 0$ (and hence $\alpha \rightarrow 1$ ), we get $|x|<1$. Therefore, $\bar{U}_{X}^{\text {sif }} \subseteq U_{X}$.

Definition 2.6. A subset $S$ of an IFNS $(X, \mu, v, *, \diamond)$ is said to be dense in $(X, \mu, v, *, \diamond)$ if and only if $\bar{S}^{(\text {sif) }}\left(\bar{S}^{(\text {wif })}\right)=X$.

Definition 2.7. An IFNS $(X, \mu, v, *, \diamond)$ is said to separable if it contains a countable dense subset, that is, there is a countable set $\left\{x_{k}\right\}$ with the following property: for each $\epsilon>0$ and each $x \in X$, there is at least one $x_{n}$ with

$$
\mu\left(x_{n}-x, \epsilon\right) \geq 1-\alpha, \quad v\left(x_{n}-x, \epsilon\right) \leq \alpha
$$

for $\alpha \in(0,1)$. 
Theorem 2.8. Every finite dimension IFNS is separable.

Proof. Let $(X, \mu, v, *, \diamond)$ be a finite dimension normed linear space and $\left\{u_{1}, u_{2}, \ldots, u_{n}\right\}$ a basis of $X$. Since $Q_{\mathbb{K}}$ is a countable subset of $\mathbb{K}$, it follows that

$$
D_{n}=\left\{\sum_{j=1}^{n} \alpha_{j} u_{j}: \alpha_{j} \in Q_{\mathbb{K}}\right\}
$$

is countable subset of $\operatorname{span}\left\{u_{1}, u_{2}, \ldots, u_{n}\right\}=X$. Also, $D$ is dense in $(X, \mu, v, *, \diamond)$. To see this, let $x \in X$ and $\epsilon>0$. Let $\beta_{1}, \ldots, \beta_{n}$ be scalars such that $x=\sum_{j=1}^{n} \beta_{j} u_{j}$. By the denseness of $Q_{\mathbb{K}}$ in $\mathbb{K}$, there exist $\alpha_{1}, \alpha_{2}, \ldots, \alpha_{n}$ in $Q_{\mathbb{K}}$ such that

$$
\mu\left(\beta_{j}-\alpha_{j}, \frac{\epsilon}{\left|\sum_{j=1}^{n} u_{j}\right|}\right) \geq 1-\alpha, \quad v\left(\beta_{j}-\alpha_{j}, \frac{\epsilon}{\left|\sum_{k=1}^{n} u_{j}\right|}\right) \leq \alpha,
$$

for all $j \in\{1,2, \ldots, n\}$. Then it follows that

$$
\mu\left(x-\sum_{j=1}^{n} \alpha_{j} u_{j}, \epsilon\right) \geq \mu\left(\left(\beta_{j}-\alpha_{j}\right) \sum_{j=1}^{n} u_{j}, \epsilon\right)=\mu\left(\beta_{j}-\alpha_{j}, \frac{\epsilon}{\left|\sum_{j=1}^{n} u_{j}\right|}\right) \geq 1-\alpha
$$

Similarly,

$$
v\left(x-\sum_{j=1}^{n} \alpha_{j} u_{j}, \epsilon\right) \leq \alpha
$$

This implies that $D$ is dense in $(X, \mu, v, *, \diamond)$.

Theorem 2.9. Every IFNS having wif-basis is separable.

Proof. Let $Y$ be IFNS with wif-basis $\left\{u_{1}, u_{2}, \ldots\right\}$. Since $Y=\bigcup_{n=1}^{\infty} Y_{n}$ with $Y_{n}=\operatorname{span}\left\{u_{1}, u_{2}, \ldots\right.$, $u_{n}$ \} for all $n \in \mathbb{N}$ is dense in $Y$, it is enough to show that $Y$ has a countable dense subset. Let

$$
D_{n}=\left\{\sum_{j=1}^{n} \alpha_{j} u_{j}: \alpha_{j} \in Q_{\mathbb{K}}\right\},
$$

for all $n \in \mathbb{N}$. Then $D_{n}$ will be a countable dense subset of $Y_{n}$ (see Theorem 2.8). Thus $\bigcup_{n=1}^{\infty} D_{n}$ is a countable dense subset of $Y$.

\section{Intuitionistic Fuzzy Schauder Bases}

In this section, we define strong and weak intuitionistic fuzzy Schauder bases. 
Definition 3.1. Let $(X, \mu, v, *, \diamond)$ be an IFNS. Then, a sequence $\left(x_{n}\right)$ is said to be

(i) strongly intuitionistic fuzzy (Schauder) basis (for short, sif-basis) of $X$ if and only if for every $x \in X$ there exists a unique sequence $\left(a_{n}\right)$ of scalars such that

$$
\sum_{k=1}^{n} a_{k} x_{k} \stackrel{\text { sif }}{\longrightarrow} x
$$

this means that for each $\alpha \in(0,1)$ there exists $n_{\circ}=n_{\circ}(\alpha)$ such that $n \geq n_{\circ}$ implies

$$
\mu\left(x-\sum_{k=1}^{n} a_{k} x_{k}, t\right) \geq 1-\alpha, \quad v\left(x-\sum_{k=1}^{n} a_{k} x_{k}, t\right) \leq \alpha,
$$

for all $t>0$,

(ii) weak intuitionistic fuzzy (Schauder) basis (for short, wif-basis) of $X$ if and only if for every $x \in X$ there exists a unique sequence $\left(a_{n}\right)$ of scalars such that

$$
\sum_{k=1}^{n} a_{k} x_{k} \stackrel{\text { wif }}{\longrightarrow} x
$$

this means, for each $\alpha \in(0,1)$ and $\epsilon>0$, there exists $n_{\circ}=n_{\circ}(\alpha, \epsilon)$ such that $n \geq n_{\circ}$ implies

$$
\mu\left(x-\sum_{k=1}^{n} a_{k} x_{k}, \epsilon\right) \geq 1-\alpha, \quad v\left(x-\sum_{k=1}^{n} a_{k} x_{k}, \epsilon\right) \leq \alpha .
$$

Proposition 3.2. Let $\left(x_{k}\right)$ be a sequence in the IFNS $(X, \mu, v, *, \diamond)$ satisfying (1.2). Then

(i) $\left(x_{k}\right)$ is a wif-basis of $X$ if and only if for every $x \in X$ there exists a unique sequence $\left(a_{n}\right)$ of scalars such that for each $\alpha \in(0,1)$,

$$
\lim _{n \rightarrow \infty}\left\|x-\sum_{k=1}^{n} a_{k} x_{k}\right\|_{\alpha}=0
$$

(ii) $\left(x_{k}\right)$ is a sif-basis of $X$ if and only if for every $x \in X$ there exists a unique sequence $\left(a_{n}\right)$ of scalars such that

$$
\lim _{n \rightarrow \infty}\left\|x-\sum_{k=1}^{n} a_{k} x_{k}\right\|_{\alpha}=0 \quad \text { uniformly in } \alpha,
$$

where $\|\cdot\|_{\alpha}$ are $\alpha$-norms of the intuitionistic fuzzy norms $(\mu, v)$.

The proof of the above theorem is similar to Propositions 2.2. 
Definition 3.3. By the notations in Definition 3.1., the mappings

$$
\begin{gathered}
g_{n}: X \longrightarrow \mathbb{R} \quad \text { such that } g_{n}(x)=g_{n}\left(\sum_{k=1}^{\infty} a_{k} x_{k}\right)=a_{n}, \\
h_{n}: X \longrightarrow X \text { such that } h_{n}(x)=h_{n}\left(\sum_{k=1}^{\infty} a_{k} x_{k}\right)=\sum_{k=1}^{n} a_{k} x_{k}, \quad n=1,2, \ldots
\end{gathered}
$$

are called coordinate functionals and natural projections, respectively, associated to the sif(wif)-basis $\left(x_{n}\right)$ in $X$.

Proposition 3.4. Let $\left(x_{n}\right)$ be a basis in wif-complete IFNS $(X, \mu, v, *, \diamond)$ satisfying (1.2). Then each $g_{n}$ and $h_{n}$ is wif-continuous.

Proof. By Proposition 3.2, $\left(x_{n}\right)$ is also a Schauder basis in the Banach space $\left(X,\|\cdot\|_{\alpha}\right)$ for each $\alpha \in(0,1)$. Thus

$$
\begin{gathered}
g_{n}:\left(X,\|\cdot\|_{\alpha}\right) \longrightarrow \mathbb{R} \quad \text { such that } g_{n}(x)=g_{n}\left(\sum_{k=1}^{\infty} a_{k} x_{k}\right)=a_{n} \\
h_{n}:\left(X,\|\cdot\|_{\alpha}\right) \longrightarrow\left(X,\|\cdot\|_{\alpha}\right) \quad \text { such that } h_{n}(x)=h_{n}\left(\sum_{k=1}^{\infty} a_{k} x_{k}\right)=\sum_{k=1}^{n} a_{k} x_{k}
\end{gathered}
$$

are continuous. Therefore, the mappings are wif-continuous for each $n$.

Remark 3.5. It is obvious that, if $\left(x_{n}\right)$ is an sif-basis of $X$ then it is wif-basis of $X$, but not conversely. For the converse part, let us consider the following example.

Example 3.6. Let $X=\ell_{p}(1 \leq p<\infty)$, the Banach space of all absolutely $p$-summable sequences with the norm $\|x\|_{p}=\left(\sum_{k}\left|x_{k}\right|^{p}\right)^{1 / p}$, and consider the intuitionistic fuzzy norm

$$
\mu(x, t)=\left\{\begin{array}{ll}
\frac{t-\|x\|_{p}}{t+\|x\|_{p}} & \text { if } t>\|x\|_{p^{\prime}} \\
0 & \text { if } t \leq\|x\|_{p^{\prime}}
\end{array} \quad v(x, t)= \begin{cases}\frac{2\|x\|_{p}}{t+\|x\|_{p}} & \text { if } t>\|x\|_{p^{\prime}} \\
1 & \text { if } t \leq\|x\|_{p}\end{cases}\right.
$$

on $X$. We can find $\alpha$-norms of intuitionistic fuzzy norm $(\mu, v)$ since it satisfies condition (1.2). Thus

$$
\begin{gathered}
\mu(x, t) \geq \alpha \Longleftrightarrow \frac{t-\|x\|_{p}}{t+\|x\|_{p}} \geq \alpha \Longleftrightarrow \frac{1+\alpha}{1-\alpha}\|x\|_{p} \leq t, \\
v(x, t) \leq 1-\alpha \Longleftrightarrow \frac{2\|x\|_{p}}{t+\|x\|_{p}} \leq 1-\alpha \Longleftrightarrow \frac{1+\alpha}{1-\alpha}\|x\|_{p} \leq t .
\end{gathered}
$$


This shows that

$$
\|x\|_{\alpha}=\inf \{t>0: \mu(x, t) \geq \alpha, v(x, t) \leq 1-\alpha\}=\frac{1+\alpha}{1-\alpha}\|x\|_{p}
$$

Now, we show that the sequence $\left(e_{k}\right)_{k=1}^{\infty}$ is wif-basis but not sif-basis. Take any $x=\left(x_{k}\right) \in \ell_{p}$. Put

$$
y_{n}=x-\left(x_{1}, x_{2}, \ldots, x_{n}, 0,0, \ldots\right)
$$

then

$$
y_{n}=\left(0,0, \ldots, x_{n+1}, x_{n+2}, \ldots\right)
$$

Hence

$$
\lim _{n}\left\|y_{n}\right\|_{\alpha}=\frac{1+\alpha}{1-\alpha} \lim _{n}\left\|y_{n}\right\|_{p}=\frac{1+\alpha}{1-\alpha} \lim _{n}\left(\sum_{k=n+1}^{\infty}\left|x_{k}\right|^{p}\right)^{1 / p}=0
$$

and by Proposition $3.2\left(e_{k}\right)$ is wif-basis for $\ell_{p}$. However, this convergence is not uniform in $\alpha$ since $(1+\alpha) /(1-\alpha) \epsilon \rightarrow \infty$ as $\alpha \rightarrow 1$.

However, if we put

$$
\mu_{1}(x, t)=\left\{\begin{array}{ll}
1 & \text { if } t>\|x\|_{p}, \\
0 & \text { if } t \leq\|x\|_{p} ;
\end{array} \quad v_{1}(x, t)= \begin{cases}0 & \text { if } t>\|x\|_{p} \\
1 & \text { if } t \leq\|x\|_{p}\end{cases}\right.
$$

on $X$, then $\left(\ell_{p}, \mu_{1}, \nu_{1}, *, \diamond\right)$ is an IFNS satisfying (1.2), and $\left(e_{k}\right)$ is a sif-basis for $\ell_{p}$ since $\|x\|_{\alpha}=$ $\|x\|_{p}$ for each $\alpha \in(0,1)$.

Remark 3.7. In finite-dimensional spaces, the definition of basis is independent of the intuitionistic fuzzy norm and hence coincides with the definition of a classical vector space basis (Hamel basis).

We know that every intuitionistic fuzzy normed space induces a topology $\tau$ such that for some $A \subset X, A \in \tau$ if and only if for each $x \in A$ there exists some $t>0$ and $\alpha \in(0,1)$ such that $B(x, \alpha, t) \subset A$, where

$$
B(x, \alpha, t):=\{y: \mu(x-y, t) \geq 1-\alpha, v(x-y, t) \leq \alpha\}
$$

Proposition 3.8. $\tau$ is a vector topology for $X$; that is, the vector space operations are continuous in this topology. 
Proof. Since the family $B(x, 1 / n, 1 / n): n=1,2, \ldots$ is a countable local basis at $x, \tau$ is the first countable topology of $X$. Hence it is sufficient to show that the vector space operations are sequentially continuous in $\tau$. Suppose $x_{n} \rightarrow x$ and $y_{n} \rightarrow y$ in the topological space $(X, \tau)$. This means $\mu\left(x_{n}-x, t / 2\right), \mu\left(y_{n}-y, t / 2\right), v\left(x_{n}-x, t / 2\right)$, and $v\left(y_{n}-y, t / 2\right) \rightarrow 1$ as $n \rightarrow \infty$, for all $t>0$. Now

$$
\begin{aligned}
& \mu\left(x_{n}+y_{n}-(x+y), t\right) \geq \mu\left(x_{n}-x, \frac{t}{2}\right) * \mu\left(y_{n}-y, \frac{t}{2}\right) \longrightarrow 1 \text { as } n \longrightarrow \infty \\
& v\left(x_{n}+y_{n}-(x+y), t\right) \leq v\left(x_{n}-x, \frac{t}{2}\right) \diamond v\left(y_{n}-y, \frac{t}{2}\right) \longrightarrow 1 \quad \text { as } n \longrightarrow \infty
\end{aligned}
$$

for all $t>0$. Further, if $\lambda_{n} \rightarrow \lambda$ in $\mathbb{R}$ or $\mathbb{C}$, the scalar field of $X$, then

$$
\begin{aligned}
\mu\left(\lambda_{n} x_{n}-\lambda x, t\right) & =\mu\left(\lambda_{n} x_{n}-\lambda x_{n}+\lambda x_{n}-\lambda x, t\right)=\mu\left(\left(\lambda_{n}-\lambda\right) x_{n}+\lambda\left(x_{n}-x\right), t\right) \\
& \geq \mu\left(x_{n}, \frac{t}{2\left|\lambda_{n}-\lambda\right|}\right) * \mu\left(x_{n}-x, \frac{t}{2|\lambda|}\right) \longrightarrow 1 \quad \text { as } n \longrightarrow \infty, \\
v\left(\lambda_{n} x_{n}-\lambda x, t\right) & =v\left(\lambda_{n} x_{n}-\lambda x_{n}+\lambda x_{n}-\lambda x, t\right)=v\left(\left(\lambda_{n}-\lambda\right) x_{n}+\lambda\left(x_{n}-x\right), t\right) \\
& \leq v\left(x_{n}, \frac{t}{2\left|\lambda_{n}-\lambda\right|}\right) \diamond v\left(x_{n}-x, \frac{t}{2|\lambda|}\right) \longrightarrow 1 \quad \text { as } n \longrightarrow \infty .
\end{aligned}
$$

Analogous to the classical results, we prove here that a normed linear space having a Schauder basis is separable.

Theorem 3.9. Let $(X, \mu, v, *, \diamond)$ be an IFNS having wif-basis $\left(x_{n}\right)$. Then the topological space $(X, \tau)$ is separable.

Proof. Let $E$ denotes the set of all finite linear combinations $\sum_{k=1}^{n} b_{k} x_{k}$, where each $b_{k}$ is a (real or complex) rational number. Obviously, $E$ is countable and let us show that it is dense in $\tau$. Suppose $x \in X$ is arbitrary. There exists a unique sequence $\left(a_{n}\right)$ of scalars such that for each $\epsilon>0$ and $\alpha \in(0,1)$, we can find some integer $n_{\circ}=n_{\circ}(\alpha, \epsilon)$ such that

$$
\mu\left(x-\sum_{k=1}^{n} a_{k} x_{k}, \epsilon\right) \geq 1-\alpha, \quad v\left(x-\sum_{k=1}^{n} a_{k} x_{k}, \epsilon\right) \leq \alpha .
$$

That is, for all $n \geq n_{\circ}$,

$$
\sum_{k=1}^{n} a_{k} x_{k} \in B(x, \alpha, \epsilon)
$$

On the other hand, one can constitute a sequence $\left(b_{k}^{i}\right)_{i=1}^{\infty}$ of scalars converging to $a_{k}$, for each $k$. Hence the sequence $\left(\sum_{k=1}^{n} b_{k}^{i} x_{k}\right)_{i=1}^{\infty}$ converges to $\sum_{k=1}^{n} a_{k} x_{k}$ in $\tau$ by the continuity of vector space operations. This implies that every $x$-centered $\tau$-open sphere $B(x, \alpha, \epsilon)$ includes an element $\sum_{k=1}^{n} b_{k}^{i} x_{k}$ of $E$. 
Theorem 3.10. Let $(X,\|\cdot\|)$ be a normed space and $\left(x_{n}\right)$ a basis in $X$. Then $\left(x_{n}\right)$ is a wif-basis for IFNS $(X, \mu, v, *, \diamond)$, where

$$
\mu(x, t)=\left\{\begin{array}{ll}
\frac{t}{t+\|x\|} & \text { if } t>0, \\
0 & \text { if } t \leq 0 ;
\end{array} \quad v(x, t)= \begin{cases}\frac{\|x\|}{t+\|x\|} & \text { if } t>0 \\
1 & \text { if } t \leq 0\end{cases}\right.
$$

Proof. By the hypothesis, for each $x \in X$, there exists a unique sequence $\left(a_{n}\right)$ of scalars with $\sum_{k=1}^{n} a_{k} x_{k} \rightarrow 0$ in the norm topology as $n \rightarrow \infty$. Explicitly, for each $\delta>0$, there exists an integer $n_{\circ}=n_{\circ}(\delta)$ such that $n \geq n_{\circ}$ implies

$$
\left\|x-\sum_{k=1}^{n} a_{k} x_{k}\right\| \leq \delta
$$

Now, for each $\epsilon$ and $\alpha \in(0,1)$, take $\delta=\alpha \epsilon /(1-\alpha)$. So, there exists an integer $n_{\circ}=n_{\circ}(\delta)=$ $n_{\circ}(\alpha, \epsilon)$ such that $n \geq n_{\circ}$ implies

$$
\left\|x-\sum_{k=1}^{n} a_{k} x_{k}\right\| \leq \frac{\alpha \epsilon}{1-\alpha}
$$

if and only if

$$
\begin{gathered}
\mu\left(x-\sum_{k=1}^{n} a_{k} x_{k}, \epsilon\right)=\frac{\epsilon}{\epsilon+\left\|x-\sum_{k=1}^{n} a_{k} x_{k}\right\|} \geq 1-\alpha, \\
v\left(x-\sum_{k=1}^{n} a_{k} x_{k}, \epsilon\right)=\frac{\epsilon}{\epsilon+\left\|x-\sum_{k=1}^{n} a_{k} x_{k}\right\|} \leq \alpha .
\end{gathered}
$$

\section{Intuitionistic Fuzzy Approximation Property}

In this section, we define strong and weak intuitionistic fuzzy approximation property and prove some interesting results.

Definition 4.1. We say that sif-complete IFNS $(X, \mu, v, *, \diamond)$ is said to have strong intuitionistic fuzzy approximation property (for short, sif-AP) if for every sif-compact set $K \subset X$ and $\alpha \in(0,1)$ there exists an operator $T: X \rightarrow X$ of finite rank such that

$$
\mu(T(x)-x, t) \geq 1-\alpha, \quad v(T(x)-x, t) \leq \alpha,
$$

for all $x \in K$ and $t>0$. 
Definition 4.2. A wif-complete IFNS $(X, \mu, v, *, \diamond)$ is said to have weak intuitionistic fuzzy approximation property (for short, wif-AP) if for every wif-compact set $K \subset X$ and for each $\epsilon>0$ and $\alpha \in(0,1)$ there exists an operator $T_{\alpha}: X \rightarrow X$ of finite rank such that

$$
\mu\left(T_{\alpha}(x)-x, \epsilon\right) \geq 1-\alpha, \quad v\left(T_{\alpha}(x)-x, \epsilon\right) \leq \alpha,
$$

for all $x \in K$.

Remark 4.3. The operator $T$ in wif-AP depends both on $\epsilon>0$ and $\alpha \in(0,1)$ whereas it depends only on $\alpha \in(0,1)$ in sif-AP. $T$ depends on the set $K$ in both situations.

Proposition 4.4. (i) A wif-complete IFNS $(X, \mu, v, *, \diamond)$ satisfying (1.2) has wif-AP if and only if for every wif-compact set $K \in X$ and for each $\epsilon>0$ and $\alpha \in(0,1)$ there exists an operator $T_{\alpha}: X \rightarrow X$ of finite rank such that

$$
\left\|T_{\alpha}(x)-x\right\|_{\alpha}<\epsilon
$$

for all $x \in K$.

(ii) A sif-complete IFNS $(X, \mu, v, *, \diamond)$ satisfying (1.2) has sif-AP if and only if for every sifcompact set $K \in X$ and for each $\epsilon>0$ there exists an operator $T: X \rightarrow X$ of finite rank such that

$$
\|T(x)-x\|_{\alpha}<\epsilon,
$$

for all $x \in K$.

The proof of the above theorem directly follows from Propositions 2.2.

Theorem 4.5. Let $(X, \mu, v, *, \diamond)$ be an IFNS possessing a wif-basis $\left(x_{n}\right)$. Then $X$ has the wif-AP.

Proof. Let $K \subset X$ be a wif-compact subset of $X$. Let $\epsilon>0$ and $\alpha \in(0,1)$ be arbitrary. By the hypothesis, for some $x \in K$, there exists a unique sequence $\left(a_{n}\right)$ of scalars such that

$$
P_{n}(x)=\sum_{k=1}^{n} a_{k} x_{k} \stackrel{\text { wif }}{\longrightarrow} x \quad \text { as } n \longrightarrow \infty .
$$

Then, there exists some $n_{\circ}(\alpha, \epsilon)$ such that

$$
\mu\left(P_{n}(x)-x, \epsilon\right) \geq 1-\alpha, \quad v\left(P_{n}(x)-x, \epsilon\right) \leq \alpha
$$

for all $n \geq n_{\circ}$. Further, each $P_{n}$ has a finite rank in the linear space $X$ since $\operatorname{dim} P_{n}(x)=$ $n$. Hence, each $P_{n}$ such that $n \geq n_{\circ}$ can be taken as a desired finite rank operator in the definition.

Remark 4.6. Theorem 4.5 can also proved for sif-basis. 
Example 4.7. Let $X=\ell_{\infty}$, the Banach space of all bounded sequence with sup-norm $\|x\|_{\infty}=$ $\sup _{n}\left|x_{n}\right|$. Also, $\|x\|_{\circ}=\sup _{n}\left|x_{n} / n\right|$ is another norm on $\ell_{\infty}$. Define the function

$$
\mu(x, t)=\left\{\begin{array}{ll}
1 & \text { if } t>\|x\|_{\infty}, \\
\frac{1}{2} & \text { if }\|x\|_{\circ}<t \leq\|x\|_{\infty}, \\
0 & \text { if } t \leq\|x\|_{0} ;
\end{array} \quad v(x, t)= \begin{cases}0 & \text { if } t>\|x\|_{\infty}, \\
\frac{1}{2} & \text { if }\|x\|_{\infty} \leq t<\|x\|_{0}, \\
1 & \text { if } t \leq\|x\|_{0} .\end{cases}\right.
$$

Then $(\mu, v)$ is an intuitionistic fuzzy norm on $\ell_{\infty}$. We can find $\alpha$-norms of intuitionistic fuzzy norm $(\mu, v)$ since it satisfies (1.2) condition. Thus

$$
\begin{gathered}
\|x\|_{\alpha}=\|x\|_{\infty} \quad \text { for } 1>\alpha>\frac{1}{2} \\
\|x\|_{\alpha}=\|x\|_{\circ} \quad \text { for } 1<\alpha \leq \frac{1}{2} .
\end{gathered}
$$

IFNS $\left(\ell_{\infty}, \mu, v, *, \diamond\right)$ cannot have a wif and hence a sif-basis since $\left(\ell_{\infty},\|\cdot\|_{\alpha}\right)=\left(\ell_{\infty},\|\cdot\|_{\infty}\right)$ for $1>\alpha>1 / 2$ and the Banach space $\left(\ell_{\infty},\|\cdot\|_{\infty}\right)$ is not separable. However, $\left(\ell_{\infty}, \mu, v, *, \diamond\right)$ has sif-AP. Recall that the set $D$ of all partitions $p=\left(\beta_{1}, \beta_{2}, \ldots, \beta_{n}\right)$ of natural numbers is a directed set by the relation $p_{a} \ll p_{b}$ which means that each $\beta_{i} \in p_{a}$ is included in some $\beta_{j}^{\prime} \in p_{b}$. Now, for each $p \in D$

$$
\Lambda_{p}(x)=\sum_{i=1}^{n} x_{h_{i}} X_{\beta_{i}}
$$

where $h_{i}$ is the distinguished point in $\beta_{i}$ and $\chi_{\beta_{i}}$ is the characteristic function of $\beta_{i}$ for $1 \leq i \leq n$. Then $\Lambda_{p}$ is a projection on $\ell_{\infty}$ of finite rank. It is well known that the set $\left(\Lambda_{p}(x), D\right)$ converges to $x$ in $\left(\ell_{\infty},\|\cdot\|_{\infty}\right)$. Let $K \subseteq \ell_{\infty}$ be sif-compact. Given $\epsilon>0$ and $x \in K$, then there exists a partition $p_{\circ}(\epsilon)$ such that for $p_{\circ}(\epsilon) \ll p$

$$
\left\|\Lambda_{p}(x)-x\right\|_{\infty}<\epsilon
$$

But

$$
\left\|\Lambda_{p}(x)-x\right\|_{\circ}<\epsilon
$$

for $p_{\circ}(\epsilon) \ll p$ since $\|x\|_{\circ} \leq\|x\|_{\infty}$ for every $x \in \ell_{\infty}$. That is, for $p_{\circ}(\epsilon) \ll p$,

$$
\left\|\Lambda_{p}(x)-x\right\|_{\alpha}<\epsilon
$$

for all $\alpha \in(0,1)$. Hence for some $\Lambda_{p}\left(p_{\circ} \ll p\right)$ meets all requirements for sif-AP in Proposition 4.4. 


\section{Acknowledgment}

This paper was completed when the first author (M. Mursaleen) visited the Yildiz Technical University, Istanbul, during June 20-July 19, 2010 under the project supported by TUBITAK BIDEB.

\section{References}

[1] J. Schauder, "Zur Theorie stetiger Abbildungen in Funktionalräumen," Mathematische Zeitschrift, vol. 26, no. 1, pp. 47-65, 1927.

[2] R. Saadati and J. H. Park, "On the intuitionistic fuzzy topological spaces," Chaos, Solitons E Fractals, vol. 27, no. 2, pp. 331-344, 2006.

[3] M. Mursaleen and S. A. Mohiuddine, "Nonlinear operators between intuitionistic fuzzy normed spaces and Fréchet derivative," Chaos, Solitons E Fractals, vol. 42, no. 2, pp. 1010-1015, 2009.

[4] M. Mursaleen and S. A. Mohiuddine, "On lacunary statistical convergence with respect to the intuitionistic fuzzy normed space," Journal of Computational and Applied Mathematics, vol. 233, no. 2, pp. 142-149, 2009.

[5] M. Mursaleen, S. A. Mohiuddine, and O. H. H. Edely, "On the ideal convergence of double sequences in intuitionistic fuzzy normed spaces," Computers \& Mathematics with Applications, vol. 59, no. 2, pp. 603-611, 2010.

[6] A. Palomares, M. Pasadas, V. Ramírez, and M. Ruiz Galán, "Schauder bases in Banach spaces: application to numerical solutions of differential equations," Computers \& Mathematics with Applications, vol. 44, no. 5-6, pp. 619-622, 2002.

[7] Y. Y1lmaz, "Schauder bases and approximation property in fuzzy normed spaces," Computers $\mathcal{E}$ Mathematics with Applications, vol. 59, no. 6, pp. 1957-1964, 2010. 


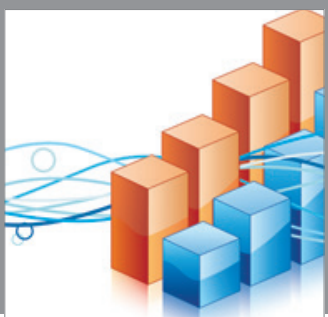

Advances in

Operations Research

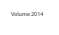



\section{The Scientific} World Journal
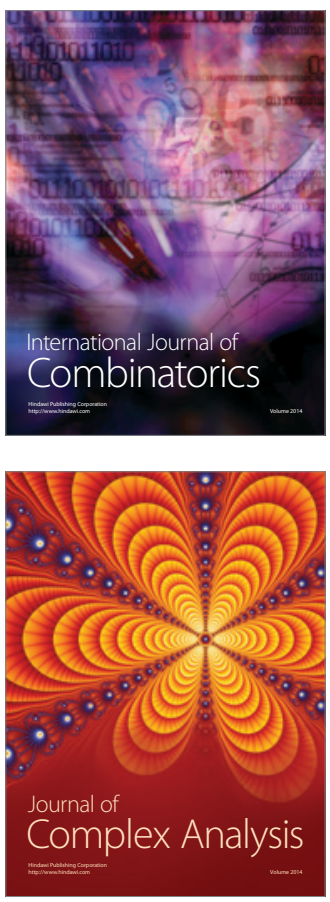

International Journal of

Mathematics and

Mathematical

Sciences
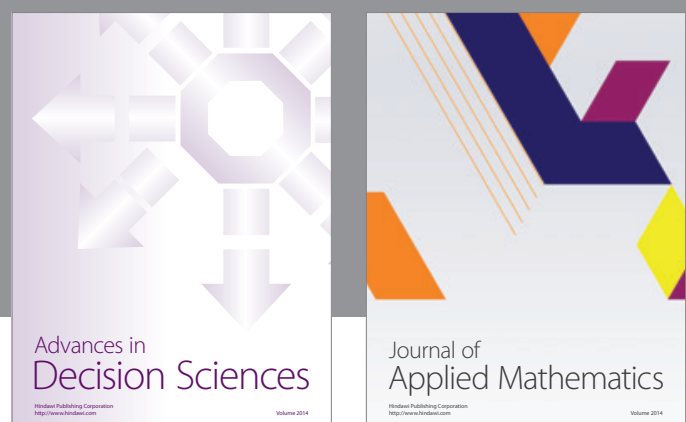

Journal of

Applied Mathematics
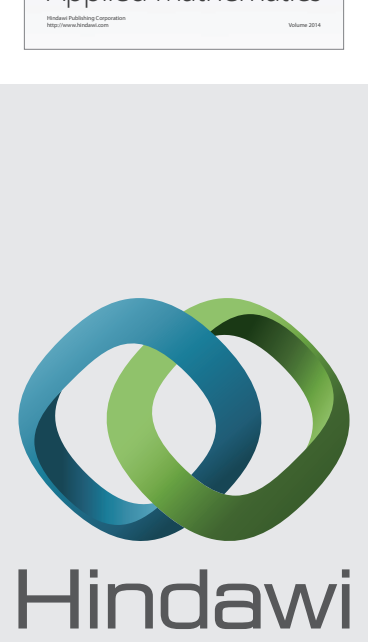

Submit your manuscripts at http://www.hindawi.com
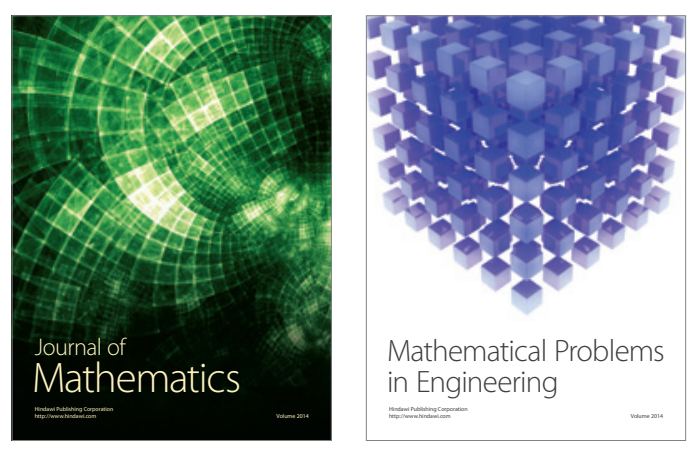

Mathematical Problems in Engineering
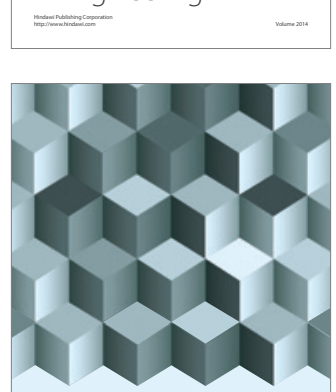

Journal of

Function Spaces
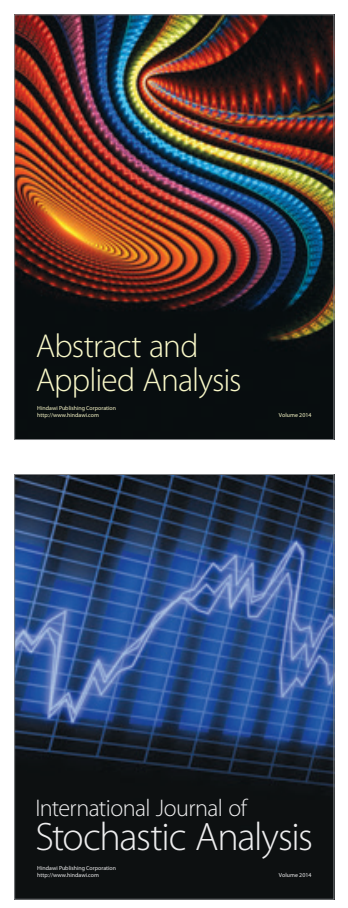

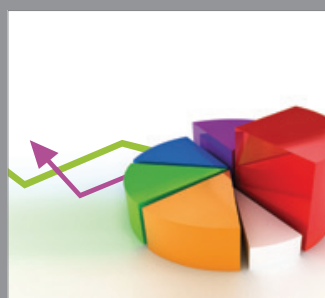

ournal of

Probability and Statistics

Promensencen
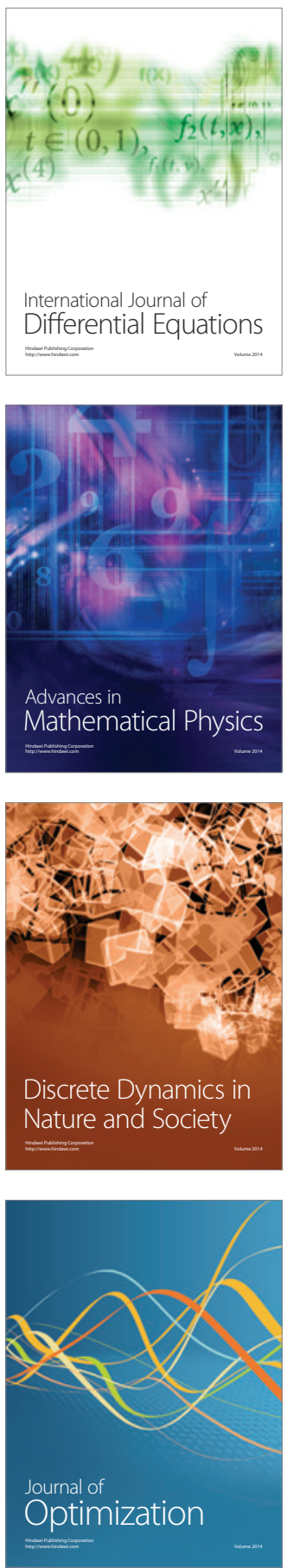